\title{
Single-color pump-probe setup at the NovoFEL facility for measurements of carrier relaxation dynamics in semiconductors
}

\author{
$\underline{\text { V. D. Kukotenko }}{ }^{1,3}$, Y. Y. Choporova ${ }^{1,2}$, Boris A. Knyazev ${ }^{1,2}$,V. V. Gerasimov ${ }^{1}$, R. K. \\ Zhukavin $^{4}$, K. A. Kovalevsky ${ }^{4}$ \\ ${ }^{1}$ Budker Institute of Nuclear Physics, Novosibirsk, Russia, kukotenkovaleria@gmail.com \\ ${ }^{2}$ Novosibirsk State University, Novosibirsk, Russia \\ ${ }^{3}$ Novosibirsk State Technical University, Novosibirsk, Russia \\ ${ }^{4}$ Institute for Physics of Microstructures, Nizhny Novgorod, Russia
}

The understanding of carrier dynamics in semiconductors is of prime importance for applications but also interesting from a fundamental point of view. As a basic example the speed of semiconductor-based photodetectors is determined by the capture time of free charge carriers. When a semiconductor like Si or $\mathrm{Ge}$ is doped with shallow impurities ( $\mathrm{As}, \mathrm{Ga}, \mathrm{Sb}$ ) the excess electrons or holes behave almost as in a hydrogen atom. However, Bohr radii and energy levels are scaled according to the effective masses and dielectric constants of their host lattice. The perturbation from there ground state and their relaxation back to equilibrium can be directly investigated by the "pump-probe" method.

To selectively excite excitation lines a monochromatic radiation source is needed. For impurities in $\mathrm{Ge}$ and $\mathrm{Si}$ these lines are in the terahertz frequency range. Temporal information is obtained using short pulses and the time resolution is determined by the pulse duration. The Novosibirsk free-electron laser (NovoFEL) facility is ideally suited for this purpose. NovoFEL generates monochromatic radiation with a tunable wavelength in the range of $6-240 \mu \mathrm{m}$. The radiation is emitted in a train of $40-100$ picosecond pulses with a repetition rate of $5.6 \mathrm{MHz}$. The spectral width is typically $1 \%$.

The idea of the pump-probe method follows a basic principle: a pump beam with a wavelength equal to a certain energy transition excites a sample. During the passage of the pump pulses, the sample becomes partially transparent as carriers are lifted into an excited state. The relaxation back to the ground state occurs on a characteristic timescale, the lifetime. A second beam, the probe beam, measures the recovery of the transmittance in time as its optical path is changed with respect to the pump pulse.

We use a wire grid polarizer to divide the initial beam into a pump beam and a probing beam. The optical path difference between both pulses can be varied by an optical delay line up to $5 \mathrm{~ns}$. Both beams are then focused onto the sample by off-axis parabolic mirrors (Fig. 1). A sample, e.g. germanium doped with arsenic (Ge:As), is placed in a liquid-He flow cryostat. The temperature of the sample can be varied from 4-200 K. As a detector, we use two Golay cells for the probe and the pump beam. The probe signal is a function of the delay between the two beams and called the pump-probe signal.

The second golay cell in the pump beam acts as a reference signal. To increase the signal-to-noise ratio, the continuous train of the NovoFEL radiation allows us to use a synchronous detection circuit (Lock-in), typically with a frequency of $15 \mathrm{~Hz}$. This frequency is given by the maximum sensitivity of the Golay cells.

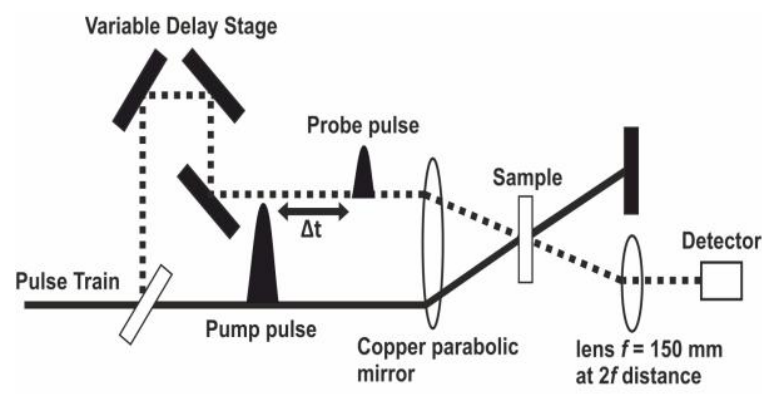

Fig. 1. Optical scheme of the "pump-probe" system at the NovoFEL.

A typical pump probe signal for a Ge:As sample is shown in figure 2 . Here, the FEL radiation was tuned to the $1 \mathrm{~s} \rightarrow 2 \mathrm{p}_{0}$ transition. The decay of the signal can be fitted by a single exponential function. The exponential time constant $\tau$ corresponds to the relaxation time of the energy transition and was found to be $\tau=1035 \pm 12 \mathrm{ps}$. The temperature was kept constant at $\mathrm{T}=5 \mathrm{~K}$, the average pump radiation power was $90 \mathrm{~mW}$, the probe radiation power was $2.5 \mathrm{~mW}$, and the wavelength was $\lambda=131.2 \mu \mathrm{m}$.

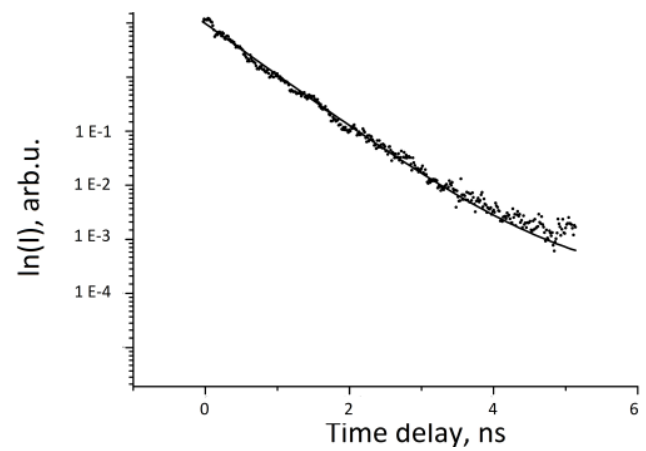

Fig. 2. Pump probe signal for a Ge:As sample at the wavelength was $\lambda=131.2 \mu \mathrm{m}$.

\section{References}

1. N. Deßmann, S. G. Pavlov. Time-resolved electronic capture in n-type germanium doped with antimony// Physical review. 2014. V. 89, No. 3. P. 1-8.

2. R. Kh. Zhukavin, K.A. Kovalevsky. The times of lowtemperature intracenter relaxation of small donors in $\mathrm{Ge} / /$ Letters to JETP. 2017. V. 789, No. 9. P. $555-560$. 